\title{
Constant-magnetic-field effect in Néel relaxation of single-domain ferromagnetic particles
}

\author{
W. T. Coffey* \\ Department of Microelectronics and Electrical Engineering, Trinity College, Dublin 2, Republic of Ireland \\ D. S. F. Crothers \\ Theoretical and Computational Physics Research Division, Department of Applied Mathematics and Theoretical Physics, \\ The Queen's University of Belfast, Belfast BT7 1NN, Northern Ireland \\ Yu. P. Kalmykov \\ Institute of Radio Engineering and Electronics, Russian Academy of Sciences, Vvedenskii Square 1, \\ Fryazino, Moscow Region 141120, Russia \\ J. T. Waldron \\ School of Computer Applications, Dublin City University, Dublin 9, Republic of Ireland \\ (Received 22 August 1994; revised manuscript received 2 February 1995)
}

\begin{abstract}
The relaxation behavior of an assembly of noninteracting single-domain ferromagnetic particles in the presence of a constant magnetic field is studied by solving the corresponding Fokker-Planck equation. The analysis is performed by first converting that equation into a hierarchy of differential-recurrence relations by expanding the solution in Legendre polynomials. The spectrum of eigenvalues and their associated amplitudes is then determined by matrix methods where all the desired physical quantities such as the magnetization correlation time and complex magnetic susceptibility may be computed numerically. In order to ensure the accuracy of the results obtained this solution is compared with an exact solution derived in terms of matrix continued fractions. It is shown that the conventional assumption in the theory of superparamagnetism, that except in the very early stages of relaxation to equilibrium the only appreciable time constant is the one associated with the smallest nonvanishing eigenvalue, is no longer true when an applied constant magnetic field exceeds a certain critical value. The breakdown of this assumption manifests itself in (a) a dramatically large deviation of the magnetization correlation time (area under the curve of the decay of the magnetization) from the inverse of the lowest eigenvalue, and (b) in the presence of relatively strong high-frequency modes superimposed on the Néel one usually assigned to the lowest eigenvalue. The results are compared with available experimental data.
\end{abstract}

\section{INTRODUCTION}

A single-domain ferromagnetic particle with uniaxial anisotropy is characterized by an internal magnetic potential which has two stable stationary points with a potential barrier between them. If the particle is sufficiently fine, the direction of the magnetization may undergo a rotation due to thermal agitation, surmounting the barrier, as described by Néel. ${ }^{1}$

The calculation of the relaxation behavior of an assembly of such particles is usually accomplished ${ }^{1-3}$ by assuming that the relaxation of the magnetization is dominated by a single relaxation mode, namely that associated with the time of reversal of the magnetization over the energy barrier between two stable orientational states. This means that in the set of eigenvalues $\left\{\lambda_{k}\right\}$ and corresponding amplitudes $\left\{A_{k}\right\}$ of the Sturm-Liouville equation (to which the Fokker-Planck equation underlying the process may be converted), $\lambda_{1}<<\lambda_{k}, k \geq 2$, and $A_{1} \gg A_{k}$ since then the decay functions $A_{k} \exp \left(-\lambda_{1} t / \tau_{N}\right), k \geq 2$ are small compared to $A_{1} \exp \left(-\lambda_{1} t / \tau_{N}\right)$ except in the very early stages of the approach to equilibrium. The diffusional relaxation time $\tau_{N}$ is defined as ${ }^{2}$

$$
\tau_{N}=\frac{v}{2 \eta k T}\left(\frac{1}{\gamma^{2}}+\eta^{2} M_{s}^{2}\right),
$$

where $\gamma$ is the gyromagnetic ratio, $M_{s}$ is the saturation magnetization, $k$ is the Bolzmann constant, $T$ is the absolute temperature, $v$ is the volume of the particle, and $\eta$ is the damping constant from Gilbert's equation, namely ${ }^{2}$

$$
\dot{\mathbf{M}}=\gamma \mathbf{M} \times\left[\mathbf{H}_{T}-\eta \dot{\mathbf{M}}\right] \text {. }
$$

In Eq. (2) $\mathbf{M}$ denotes the magnetization and

$$
\mathbf{H}_{T}=\mathbf{h}-\partial V / \partial \mathbf{M},
$$

where $\mathbf{h}$ is the random white-noise field arising from thermal agitation and $V$ is the barrier potential including that of the internal crystalline anisotropy and the applied external field $\mathbf{H}$.

In view of the above considerations the early studies ${ }^{2-4}$ of the relaxation process were confined to the calculation of the smallest nonvanishing eigenvalue of the SturmLiouville equation making the assumption that the process is dominated by a single relaxation mode with the time constant 


$$
T \approx \frac{\tau_{N}}{\lambda_{1}}
$$

Very recently, the relaxation behavior has been reexamined by Coffey et al..$^{5}$ for the simple uniaxial potential of the crystalline anisotropy

$$
V(\vartheta)=K \sin ^{2} \vartheta
$$

This is an axially symmetric bistable potential with anisotropy constant $K$ representing the free energy per unit volume of a particle. The stable configurations of the magnetization $\mathbf{M}$ are at $\vartheta=0$ and $\vartheta=\pi$ where the orientation for $\mathbf{M}$, on a sphere of radius $M_{s}$ is specified by the spherical polar coordinates $\vartheta$ and $\phi, \vartheta$ being the polar angle. The calculation of the decay of the longitudinal component of the magnetization following the removal of a weak constant applied field $\Delta \mathbf{H}$ superimposed on the field $\mathbf{H}$ then amounts to the calculation of $A_{k}$ and $\lambda_{k}$ in the equation

$$
\begin{aligned}
M_{z}(t)=m N f_{1}(t) & =m N\langle\cos \vartheta\rangle \\
& =\frac{m^{2} N \Delta H}{k T} \sum_{k} A_{k} e^{-\lambda_{k} t / \tau_{N}} .
\end{aligned}
$$

Here $N$ is the number of particles per unit volume,

$$
m=M_{s} v
$$

is the magnetic moment of a particle. According to linear-response theory the decay function $f_{1}(t)$ from Eq. (6) is connected with the equilibrium longitudinal autocorrelation function of the magnetization $C_{1}(t)$ as follows:

$$
\frac{f_{1}(t)}{f_{1}(0)}=C_{1}(t)=\frac{\langle\cos \vartheta(0) \cos \vartheta(t)\rangle_{0}-\langle\cos \vartheta(0)\rangle_{0}^{2}}{\left\langle\cos ^{2} \vartheta(0)\right\rangle_{0}-\langle\cos \vartheta\rangle_{0}^{2}},
$$

where the symbol \langle\rangle$_{0}$ means the equilibrium ensemble average, so that the magnetization correlation time $T_{\|}$ according to Eq. (6) is given by

$$
T_{\|}=\int_{0}^{\infty} C_{1}(t) d t=\tau_{N} \frac{\sum_{k} A_{k} \lambda_{k}^{-1}}{\sum_{k} A_{k}} .
$$

It should be noted that in our notation $\lambda_{k}$ differs by a factor of 2 from that used in Refs. 2-4. Coffey et al. ${ }^{5}$ derived an exact analytic equation for the correlation time $T_{\|}$. Also, by calculating successive $\lambda_{k}$ and $A_{k}$ Coffey et $a l .^{5}$ were readily able to show (cf. their Table I) that the relaxation process under the influence of the potential of Eq. (5) is accurately represented by the first decay mode. Thus, Eq. (4) holds accurately in this case (Table II of Ref. 5).

The second type of uniaxial potential which is of interest in the study of superparamagnetism is when a constant field $\mathbf{H}$ of arbitrary strength is superimposed on the anisotropy potential. ${ }^{1-4}$ In general such a field can only be applied at some angle to the easy axis of magnetization since that axis is in a random position. However, in order to preserve the axial symmetry of the problem and its attendant mathematical simplifications we shall suppose as in Refs. 1-4 that the field is applied along the polar axis so that the potential $V$ is of the form

$\frac{v V(\vartheta)}{k T}=\sigma \sin ^{2} \vartheta-\xi \cos \vartheta=\sigma\left(\sin ^{2} \vartheta-2 h \cos \vartheta\right)$,

where the barrier height parameter

$$
\sigma=\frac{K v}{k T}
$$

and the external field parameter

$$
\xi=\frac{v H M_{s}}{k T}
$$

with

$$
h=\frac{\xi}{2 \sigma} .
$$

This potential was originally introduced by $\mathrm{Né}^{1}$ who gave an expression for the time of reversal of the magnetization using the discrete orientation approximation. It was further studied by Brown ${ }^{2}$ who obtained approximate expressions for the lowest nonvanishing eigenvalue in the limit of large and small $\sigma$ using the Kramers transition state method ${ }^{6}$ and perturbation theory, respectively. Later $\lambda_{1}^{-1}$ was calculated numerically by Aharoni. ${ }^{4}$ However, he did not calculate any other $\lambda^{s}$ or the associated amplitudes including that of $\lambda_{1}^{-1}$ thus it was not possible to ascertain the role they play in the relaxation process, nor is it possible to test the accuracy of the singlemode approximation. On the other hand the analysis presented by Garanin, Ischenko, and Panina ${ }^{7}$ enabled them to derive an integral expression for the correlation time $T_{\|}$from the Sturm-Liouville equation. However, rather than trying to calculate $T_{\|}$exactly from their equation, they presented various asymptotic formulas for $T_{\|}$and the complex susceptibility.

It is the purpose of this paper to study in detail the relaxation behavior of the system under consideration. In order to accomplish this, we shall calculate accurately the eigenvalues $\left\{\lambda_{k}\right\}$ and the corresponding amplitudes $\left\{A_{k}\right\}$, the correlation time $T_{\|}$and the complex susceptibility for the potential of Eq. (10) using the methods we have described in Refs. 5 and 8-10. The most important results of the calculation are (a) that the correlation time has behavior dramatically different from $\lambda_{1}^{-1}$ above certain critical values of the parameters $\sigma$ and $h$ and (b) the existence of high-frequency relaxation modes in addition to that arising from the low-frequency one associated with the reversal of $\mathbf{M}$. This is due to the fact that the high-frequency modes make a distinct contribution to the response.

\section{DIFFERENTIAL-RECURRENCE RELATIONS FOR RELAXATION IN THE PRESENCE OF A CONSTANT FIELD}

The Fokker-Planck equation for the probability density $W(\vartheta, \phi, t)$ of orientations of the magnetization $\mathbf{M}$ on the unit sphere for an axially symmetric potential $V(\vartheta)$ is $^{2}$ 


$$
\begin{aligned}
2 \tau_{N} \frac{\partial W}{\partial t}= & \frac{1}{\sin \vartheta} \frac{\partial}{\partial \vartheta}\left[\sin \vartheta\left[\frac{\partial W}{\partial \vartheta}+\frac{v}{k T} \frac{\partial V}{\partial \vartheta} W\right]\right. \\
& +\frac{1}{\sin \vartheta} \frac{\partial}{\partial \phi}\left\{\frac{v}{k T}\left(\frac{\partial V}{\alpha \partial \vartheta}\right) W+\frac{1}{\sin \vartheta} \frac{\partial W}{\partial \phi}\right\},
\end{aligned}
$$

where

$$
\alpha=\eta \gamma M_{s}
$$

is a dimensionless damping parameter. In order to study the longitudinal relaxation behavior we suppose that the constant field term in the potential of Eq. (10) is altered by a small amount $\xi_{1}=m \Delta H / k T<<1$ at an initial time $t=0$ so that we determine the after-effect solution of Eq. (14). We can disregard the dependence of $W$ on $\phi$ for the longitudinal relaxation; hence we may assume that the distribution function $W$ is

$$
W(\vartheta, t)=\sum_{l=0}^{\infty} a_{l}(t) P_{l}(\cos \vartheta),
$$

where the $P_{l}$ are the Legendre polynomials. On substituting Eq. (16) into Eq. (15) we obtain the differentialrecurrence relation

$$
\begin{aligned}
& \frac{2 \tau_{N}}{l(l+1)} \dot{f}_{l}(t)+\left[1-\frac{2 \sigma}{(2 l-1)(2 l+3)}\right] f_{l}(t) \\
& =\frac{\xi}{2 l+1}\left[f_{l-1}(t)-f_{l+1}(t)\right] \\
& \quad+\frac{2 \sigma(l-1)}{(2 l+1)(2 l-1)} f_{l-2}(t) \\
& \quad-\frac{2 \sigma(l+2)}{(2 l+1)(2 l+3)} f_{l+2}(t)
\end{aligned}
$$

where

$f_{l}(t)=\left\langle P_{l}(\cos \vartheta)\right\rangle-\left\langle P_{l}(\cos \vartheta)\right\rangle_{0}=\frac{a_{l}(t)-a_{l}(\infty)}{(2 l+1) a_{0}}$

and the symbols \langle\rangle and \langle\rangle$_{0}$ mean ensemble average and equilibrium ensemble average, respectively.

We are interested in the decay of the magnetization $M_{z}(t)$ which in this case is

$$
\begin{aligned}
M_{z}(t) & =m N\left[\langle\cos \vartheta\rangle-\langle\cos \vartheta\rangle_{0}\right] \\
& =m N \int_{0}^{\pi}\left(\cos \vartheta-\langle\cos \vartheta\rangle_{0}\right) W(\vartheta, t) \sin \vartheta d \vartheta \\
& =m N f_{1}(t)
\end{aligned}
$$

so that we are required to calculate $f_{1}(t)$. This is considerably more involved than the case $\xi=0$ because Eq. (17) does not decouple into separate sets for even and odd $f_{1}(t)$. Furthermore, it is a five-term recurrence relation so that it is not obvious how its solution may be found in the form of a scalar continued fraction. It may however, be cast into the form of a three-term matrix recurrence relation as in Ref. 10 .

The initial conditions for $f_{l}(0)$ may be determined as follows:

$$
f_{l}(0)=\frac{\int_{-1}^{+1} e^{\sigma x^{2}+\left(\xi+\xi_{1}\right) x} P_{l}(x) d x}{\int_{-1}^{+1} e^{\sigma x^{2}+\left(\xi+\xi_{1}\right) x} d x}-\left\langle P_{l}(x)\right\rangle_{0},
$$

which in the linear approximation in the perturbation $\xi_{1}$ reduces to

$$
f_{l}(0)=\xi_{1}\left[\left\langle x P_{l}(x)\right\rangle_{0}-\langle x\rangle_{0}\left\langle P_{l}(x)\right\rangle_{0}\right]
$$

or

$$
\begin{gathered}
f_{l}(0)=\xi_{1}\left[\frac{l+1}{(2 l+1)}\left\langle P_{l+1}\right\rangle_{0}+\frac{l}{2 l+1}\left\langle P_{l-1}\right\rangle_{0}\right. \\
\left.-\left\langle P_{1}\right\rangle_{0}\left\langle P_{l}\right\rangle_{0}\right]
\end{gathered}
$$

or

$$
f_{l}(0)=\xi_{1}\left[\frac{l+1}{2 l+1} f_{l+1}^{0}+\frac{l}{2 l+1} f_{l-1}^{0}-f_{1}^{0} f_{l}^{0}\right],
$$

where

$$
f_{l}^{0}(0)=\left\langle P_{l}\right\rangle_{0}
$$

The equilibrium quantities $f_{l}^{0}$ satisfy the set of equations

$$
\begin{aligned}
{\left[1-\frac{2 \sigma}{(2 l-1)(2 l+3)}\right] f_{l}^{0}=} & \frac{\xi}{2 l+1}\left[f_{l-1}^{0}-f_{l+1}^{0}\right] \\
& +\frac{2 \sigma(l-1)}{(2 l+1)(2 l-1)} f_{l-2}^{0} \\
& -\frac{2 \sigma(l+2)}{(2 l+1)(2 l+3)} f_{l+2}^{0},
\end{aligned}
$$

which is the set of Eq. (17) with $\dot{f}_{l}=0$.

The recurrence relation of Eq. (24) can be evaluated for any $l$ if we have a knowledge of $\left\langle P_{0}\right\rangle_{0},\left\langle P_{1}\right\rangle_{0}$, and $\left\langle P_{2}\right\rangle_{0}$. These may be given just as in Ref. 10 by using the results of p. 369 of Ref. 11. We have

$$
\begin{aligned}
& \left\langle P_{0}\right\rangle_{0}=1, \\
& \left\langle P_{1}\right\rangle_{0}=\frac{\xi}{T(\xi, \sigma)}-\frac{\xi}{2 \sigma}, \\
& \left\langle P_{2}\right\rangle_{0}=\frac{3}{2}\left[\frac{\xi L(\xi)+1-\xi^{2} / 2 \sigma}{T(\xi, \sigma)}+\frac{\xi^{2} / 2 \sigma-1}{2 \sigma}\right)-\frac{1}{2},
\end{aligned}
$$

where

$$
L(\xi)=\operatorname{coth} \xi-\frac{1}{\xi}
$$

is the Langevin function and the function $T(\xi, \sigma)$ is given in terms of Dawson's integral ${ }^{11,12}$

$$
D(x)=e^{-x^{2}} \int_{0}^{x} e^{t^{2}} d t
$$

of given argument as 


$$
\begin{aligned}
T(\xi, \sigma)=\sqrt{\sigma}[ & {[\xi L(\xi)+1+\xi] D\left[\sqrt{\sigma}+\frac{\xi}{2 \sqrt{\sigma}}\right] } \\
& \left.+[\xi L(\xi)+1-\xi] D\left[\sqrt{\sigma}-\frac{\xi}{2 \sqrt{2 \sigma}}\right]\right] .
\end{aligned}
$$

The set of equations (17) may be solved to yield the relaxation behavior of $f_{1}(t)$ in either of two ways. The first is to arrange them in the form

$$
\dot{\mathbf{X}}(t)=\mathbf{A X}(t),
$$

and the second is to arrange the differential-recurrence relation as a three-term matrix one, the Laplace transform of which may then be obtained analytically in terms of a matrix continued fraction as described for the corresponding two-dimensional problem in Ref. 10 .

\section{SOLUTION OF THE SET OF RECURRENCE RELATIONS (17), FORMULATION AS A MATRIX DIFFERENTIAL EQUATION}

The first method proceeds as follows. In Eq. (30) the column vector $\mathbf{X}(t)$ is

$$
\mathbf{X}(t)=\left(\begin{array}{c}
f_{1}(t) \\
f_{2}(t) \\
\cdot \\
\cdot \\
f_{l}(t) \\
\cdot \\
\cdot
\end{array}\right)
$$

The system matrix $\mathbf{A}$ is determined by Eq. (17) and is given by

$$
\mathbf{A}=-\frac{1}{\tau_{N}}\left(\begin{array}{ccccccc}
1-\frac{2}{5} \sigma & \frac{\xi}{3} & \frac{2}{5} \sigma & 0 & 0 & 0 & \ldots \\
-\frac{3}{5} \xi & 3-\frac{2}{7} \sigma & \frac{3}{5} \xi & \frac{24}{35} \sigma & 0 & 0 & \ldots \\
-\frac{24}{35} \sigma & -\frac{6}{7} \xi & 6-\frac{4}{15} \sigma & \frac{6}{7} \xi & \frac{20}{21} \sigma & 0 & \ldots \\
0 & -\frac{20}{21} \sigma & -\frac{10}{9} \xi & 10-\frac{20}{77} \sigma & \frac{10}{9} \xi & \frac{40}{33} \sigma & \ldots \\
: & : & : & : & : & : & : \\
: & : & : & : & : & : & :
\end{array}\right)
$$

In Eq. (17), $l$ is taken large enough (equal to $L$ say) to ensure convergence of the set of Eqs. (30). The lowest nonvanishing eigenvalue which corresponds to the reciprocal of the greatest relaxation time, is then the smallest root of the characteristic equation

$$
\operatorname{det}(\lambda \mathbf{I}-\mathbf{A})=0 \text {. }
$$

The relaxation modes of $f_{1}(t)$ may be found from Eq. (30) by assuming that ${ }^{13} \mathbf{A}$ has a linearly independent set of $L$ eigenvectors $\left(\mathbf{R}_{1}, \ldots, \mathbf{R}_{L}\right)$, so that ${ }^{13,14}$

$$
\mathbf{X}(t)=b_{1} e^{\lambda_{1} t} \mathbf{R}_{1}+b_{2} e^{\lambda_{2} t} \mathbf{R}_{2} \cdots+b_{L} e^{\lambda_{L} t} \mathbf{R}_{L},
$$

where the $b_{i}$ are to be determined from the initial conditions (23). Equations (30) may now be solved to any desired degree of accuracy to yield the decay of the longitudinal component of the magnetization according to Eq. (6), namely

$$
\begin{aligned}
m N f_{1}(t) & =m N\left[\langle\cos \vartheta\rangle-\langle\cos \vartheta\rangle_{0}\right] \\
& =\frac{m^{2} \Delta H N}{k T} \sum_{k=1}^{\infty} A_{k} e^{-\lambda_{k} t / \tau_{N}} .
\end{aligned}
$$

The corresponding normalized complex susceptibility

$$
\chi_{\|}(\omega)=\chi_{\|}^{\prime \prime}(\omega)-i \chi_{\|}^{\prime \prime}(\omega)=G \alpha_{\|}(\omega),
$$

where

$$
G=\frac{m^{2} N}{3 k T},
$$

is then given by

$$
\alpha_{\|}(\omega)=\sum_{k=1}^{\infty} \frac{3 A_{k}}{1+i \omega \tau_{N} / \lambda_{k}} .
$$

Here we have made use of the linear-response theory formula $^{15}$

$$
\begin{aligned}
\alpha_{\|}(\omega) & =\alpha_{\|}^{\prime}(\omega)-i \alpha_{\|}^{\prime \prime}(\omega) \\
& =\alpha_{\|}^{\prime}(0)\left[1-i \omega \int_{0}^{\omega} \frac{f_{1}(t)}{f_{1}(0)} e^{-i \omega t} d t\right] .
\end{aligned}
$$

The correlation time $T_{\|}$in terms of the $A_{k}$ and $\lambda_{k}$ is given by Eq. (9).

We note that the correlation time $T_{\|}$itself may be evaluated more simply by noting that as in Ref. 5

$$
T_{\|}=\lim _{s \rightarrow 0} \int_{0}^{\infty} C_{1}(t) e^{-s t} d t=\widetilde{C}_{1}(0)=\frac{\widetilde{f}_{1}(0)}{f_{1}(0)}
$$

with the tilde denoting the Laplace transform where $\widetilde{f}_{1}(0)$ is determined from the matrix equation ${ }^{5}$ 


$$
\widetilde{\mathbf{X}}(0)=-\mathbf{A}^{-1} \mathbf{X}(0),
$$

where $\mathbf{X}(0)$ is the initial value vector that is

$$
\mathbf{X}(0)=\left(\begin{array}{c}
f_{1}(0) \\
f_{2}(0) \\
f_{3}(0) \\
:
\end{array}\right) .
$$

Equation (41) is obtained from the Laplace transform of Eq. (30) for $s=0$.

We have formulated the problem of determining the initial values $f_{1}(0)$ from the recurrence relation with the first three $f_{l}^{0}$ given as in Eqs. (25)-(29). The $f_{l}^{0}$ may however, be evaluated numerically by simply calculating $\mathbf{A}^{-1}$. First we note that the set of Eq. (24) constitutes the inhomogeneous set

$$
\mathbf{A F} \mathbf{F}^{0}=\mathbf{B}
$$

with

$$
\mathbf{F}^{0}=\left(\begin{array}{c}
f_{1}^{0} \\
f_{2}^{0} \\
: \\
f_{l}^{0} \\
:
\end{array}\right)
$$

and

$$
\begin{aligned}
& {\left[\begin{array}{c}
\dot{f}_{2 l-1}(t) \\
\dot{f}_{2 l}(t)
\end{array}\right]=\frac{1}{\tau_{N}}\left(\begin{array}{cc}
\frac{4 \sigma(l-1)(2 l-1) l}{(4 l-1)(4 l-3)} & \frac{\xi(2 l-1) l}{(4 l-1)} \\
0 & \frac{2 \sigma(2 l-1)(2 l+1) l}{(4 l+1)(4 l-1)}
\end{array}\right)\left(\begin{array}{l}
f_{2 l-3}(t) \\
f_{2 l-2}(t)
\end{array}\right)}
\end{aligned}
$$

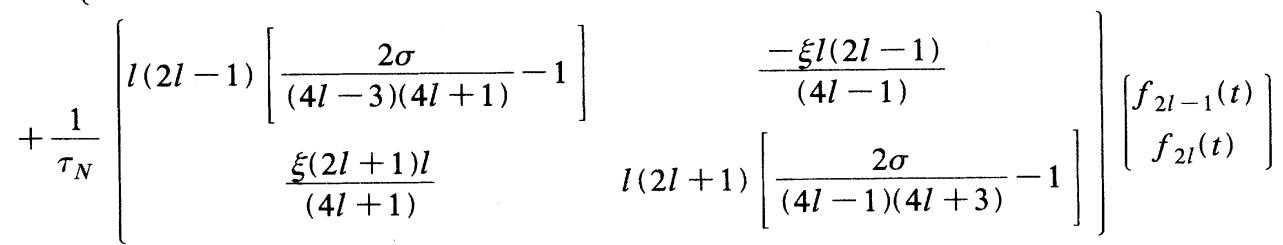

$$
\begin{aligned}
& +\frac{1}{\tau_{N}}\left(\begin{array}{cc}
\frac{-2 \sigma(2 l+1)(2 l-1) l}{(4 l-1)(4 l+1)} & 0 \\
\frac{-\xi(2 l+1) l}{(4 l+1)} & \frac{-4 \sigma(l+1)(2 l+1) l}{(4 l+1)(4 l+3)}
\end{array}\right)\left[\begin{array}{l}
f_{2 l+1}(t) \\
f_{2 l+2}(t)
\end{array}\right] \text {. }
\end{aligned}
$$

A general method of solution of Eq. (46) in terms of matrix continued fractions was given by Risken. ${ }^{13}$ Another approach which has the merit of being considerably more simple than the previously available algorithm has been described in Ref. 10. Thus Eq. (47) may be solved for the Laplace transform

$$
\widetilde{\mathbf{C}}_{1}(s)=\left[\begin{array}{l}
\widetilde{f}_{1}(s) \\
\widetilde{f}_{2}(s)
\end{array}\right]
$$

using the method of Ref. 10 to yield

$$
\widetilde{\mathbf{C}}_{1}(s)=\left[s \mathbf{I}-\mathbf{Q}_{1}^{-}-\mathbf{Q}_{1}^{+} \mathbf{S}_{2}(s)\right]^{-1}\left\{\mathbf{C}_{1}(0)+\sum_{n=2}^{\infty} \prod_{k=2}^{n} \mathbf{Q}_{k-1}^{+} \widetilde{\mathbf{S}}_{2}(s)\left(\mathbf{Q}_{k}^{-}\right)^{-1} \mathbf{C}_{n}(0)\right\},
$$


where the $2 \times 2$ matrices $\mathbf{Q}_{n}^{ \pm}, \mathbf{Q}_{n}$ are as shown in Eq. (47), $\widetilde{\mathbf{S}}_{n}(s)$ is the $2 \times 2$ matrix continued fraction defined as

$$
\widetilde{\mathbf{S}}_{n}(s)=\left(s \mathbf{I}-\mathbf{Q}_{n}-\mathbf{Q}_{n}^{+} \widetilde{\mathbf{S}}_{n+1}(s)\right)^{-1} \mathbf{Q}_{n}^{-} .
$$

The initial value vectors

$$
\mathbf{C}_{n}(0)=\left[\begin{array}{c}
f_{2 n-1}(0) \\
f_{2 n}(0)
\end{array}\right)
$$

are most conveniently determined from Eqs. (23) and (24) in this case. The after-effect function $\widetilde{f}_{1}(s)$ is simply calculated from the scalar product of $\widetilde{\mathbf{C}}_{1}(s)$ with the unit vector $\mathbf{e}=(1,0)$ namely

$$
\widetilde{f}_{1}(s)=\left[\widetilde{\mathbf{C}}_{1}(s) \cdot \mathbf{e}\right],
$$

while the correlation time $T_{\|}$and the complex susceptibility $\chi_{\|}(\omega)$ are given as before by Eqs. (40) and (39), respectively. Equations $(48)-(50)$ constitute the exact solution of the problem in terms of matrix continued fractions.

\section{RESULTS AND COMPARISON WITH EXPERIMENTAL DATA}

In Fig. 1 and Table $I$ we show the behavior of the lowest eigenvalue $\lambda_{1}$ calculated from the characteristic Eq. (33) as a function of $\sigma$ for various values of $h$ showing that our calculation agrees with the corresponding Fig. 1 of Aharoni $^{4}$ in all respects. A $45 \times 45$ matrix $A$ was sufficient to obtain a value of $\lambda_{1}^{-1}$ accurate to three significant digits for the range of parameters chosen. In Fig. 2 and Table II we show the behavior of the correlation time $T_{\|}$computed from the matrix formula Eq. (41) for the same values of $h$. The most astonishing result of this calculation is that $T_{\|}^{-1}$ differs greatly from $\lambda_{1}^{-1}$ above a certain critical value of $h$. Again a $45 \times 45$ matrix yielded satisfactory results. For example, at $h=0.2$ and $\sigma=20, \quad T_{\|}=13.6$ and $\lambda_{1}^{-1}=1.01 \times 10^{4}$. This behavior is further emphasized in Table III where one can easily see that $\lambda_{1}^{-1}$ differs dramatically from $T_{\|}$. Thus the correlation time is far more sensitive to any al-

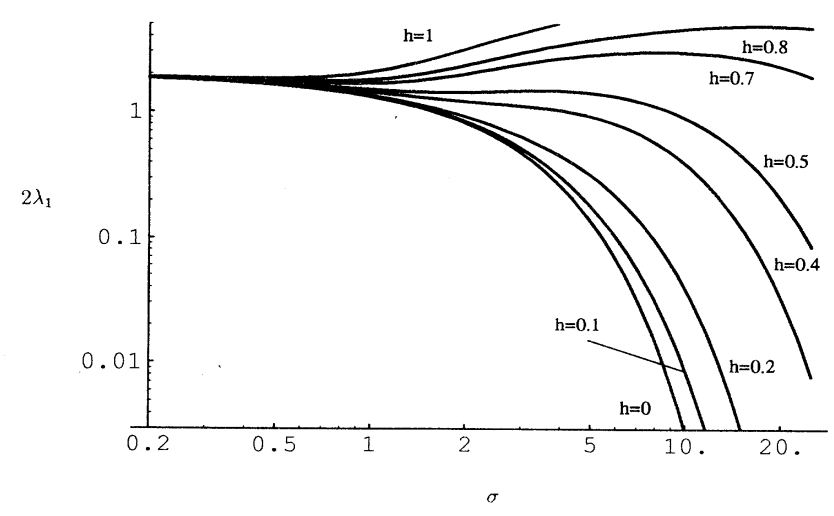

FIG. 1. The lowest nonvanishing eigenvalue $\lambda_{1}$ as a function of the barrier height parameter $\sigma$ for various values of the field parameter $h$.
TABLE I. The lowest nonvanishing eigenvalue $\lambda_{1}$ for various values of the barrier height $(\sigma)$ and field $(h)$ parameters.

\begin{tabular}{lcccllll}
\hline \hline \multicolumn{10}{c}{$h \lambda_{1}$} \\
\multicolumn{1}{c}{} & $\sigma=0.2$ & $\sigma=0.5$ & $\sigma=1$ & $\sigma=2$ & $\sigma=5$ & $\sigma=10$ & \multicolumn{1}{c}{$\sigma=20$} \\
\hline 0.01 & 1.84 & 1.63 & 1.31 & 0.808 & 0.136 & 0.00293 & $4.24 \times 10^{-7}$ \\
0.1 & 1.84 & 1.63 & 1.31 & 0.832 & 0.179 & 0.0086 & $7.79 \times 10^{-6}$ \\
0.2 & 1.85 & 1.63 & 1.34 & 0.906 & 0.313 & 0.0383 & 0.000198 \\
0.4 & 1.85 & 1.66 & 1.43 & 1.2 & 0.91 & 0.385 & 0.0327 \\
0.5 & 1.85 & 1.68 & 1.49 & 1.41 & 1.4 & 0.883 & 0.206 \\
0.7 & 1.86 & 1.72 & 1.67 & 1.97 & 2.78 & 2.93 & 2.27 \\
0.8 & 1.86 & 1.75 & 1.78 & 2.32 & 3.67 & 4.52 & 4.78 \\
1 & 1.88 & 1.82 & 2.05 & 3.12 & 5.82 & 8.78 & 12.9 \\
\hline \hline
\end{tabular}

teration of the two potential well structure of Eq. (5) caused by the imposition of the field $\xi$ than is $\lambda_{1}^{-1}$. We were first alerted to this behavior by calculating $T_{\|}$using the matrix formula of Eq. (41) which merely requires a knowledge of the initial value vector $\mathbf{X}(0)$ and $\mathbf{A}^{-1}$. More insight into the disparity between $\lambda_{1}^{-1}$ and $T_{\|}$may be gained by calculating $T_{\|}$using Eq. (9). This requires a knowledge of a sufficiently large set of the eigenvalues $\left\{\lambda_{k}\right\}$ and their corresponding amplitudes $\left\{A_{k}\right\}$ and is shown in Table IV. The reason for the disparity between $T_{\|}$and $\lambda_{1}^{-1}$ now becomes obvious. It is due to the fact that at short to intermediate times the high-frequency decay modes cannot be neglected as they contribute significantly to the correlation time. Indeed it is apparent by using the values of Table IV that Eq. (9) for the correlation time $T_{\|}$cannot be reduced to Eq. (4) in contrast to the case $\xi=0$ where the $A_{k}$ are negligible for all $k>1$ (see Table I of Ref. 5) so that

$$
\frac{\sum A_{k} \lambda_{k}^{-1}}{\sum A_{k}} \approx \frac{1}{\lambda_{1}} \text {. }
$$

The results are corroborated by the matrix continued fraction method of Sec. IV.

By way of further illustration of our results we show in Table $\mathrm{V}$ the behavior of the first ten decay modes $A_{k} \exp \left(-\lambda_{k} t / \tau_{N}\right)$ for various values of the time $t$ ranging from 0.01 to 10 . The general structure of the decay

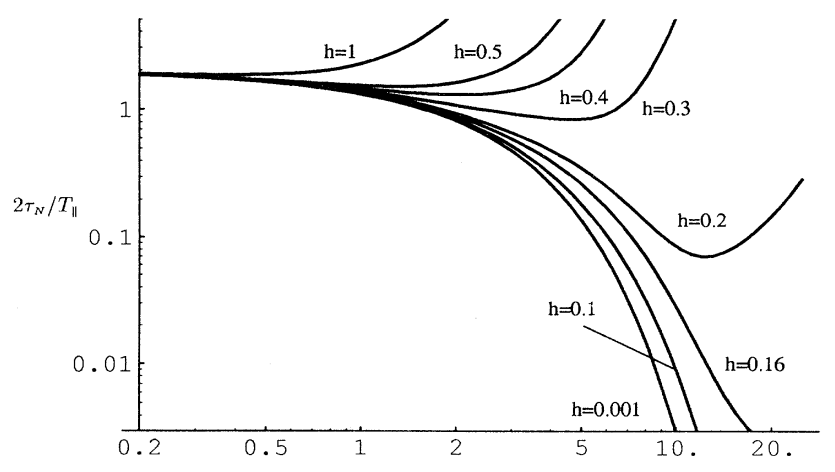

FIG. 2. Inverse of the correlation time $T_{\|}$as a function of the barrier height parameter $\sigma$ for various values of the field parameter $h$. 
TABLE II. Inverse of the correlation time $T_{\|}$for various values of the barrier height $(\sigma)$ and field $(h)$ parameters.

\begin{tabular}{lllllllll}
\hline \hline \multicolumn{10}{c}{$h$} & $\sigma=0.2$ & $\sigma=0.5$ & $\sigma=1$ & $\sigma=2$ & $\sigma=5$ & $\sigma=10$ & $\sigma=20$ \\
\hline 0.01 & 1.84 & 1.63 & 1.31 & 0.813 & 0.138 & 0.00294 & $4.24 \times 10^{-7}$ \\
0.1 & 1.84 & 1.63 & 1.32 & 0.84 & 0.183 & 0.00892 & 0.0000107 \\
0.2 & 1.85 & 1.64 & 1.34 & 0.925 & 0.35 & 0.0842 & 0.147 \\
0.4 & 1.85 & 1.66 & 1.45 & 1.29 & 2.74 & 43.3 & 104 \\
0.5 & 1.85 & 1.68 & 1.53 & 1.61 & 9.16 & 51.8 & 112 \\
0.7 & 1.86 & 1.74 & 1.74 & 2.65 & 24.6 & 60.8 & 129 \\
0.8 & 1.87 & 1.77 & 1.89 & 3.45 & 28 & 65.1 & 137 \\
1 & 1.88 & 1.85 & 2.24 & 5.73 & 33.2 & 73.7 & 154 \\
\hline \hline
\end{tabular}

modes now becomes apparent. In this case $(\sigma=10)$ for small values of $t<0.1$ the high-frequency mode characterized by $\lambda_{5}$ makes a greater contribution to the decay than that of $\lambda_{1}$ however, as time progresses (see Fig. 3) this and all other modes vanish so that only the $\lambda_{1}$ mode - which is, as usual, that associated with the stability of a given distribution of magnetization - Néel relaxation remains. It is also obvious from Fig. 3 that the general behavior of the correlation function $C_{1}(t)$ differs markedly from that of the first mode decay function $\exp \left(-\lambda_{1} t / \tau_{N}\right)$. The dominance of the high-frequency modes at short and intermediate times has important consequences in the frequency domain, where a highfrequency absorption peak will appear in the imaginary part $\chi_{\|}^{\prime \prime}(\omega)$ of the complex susceptibility along with the usual low-frequency absorption maximum associated with $\lambda_{1}$. This behavior is illustrated in Figs. 4 and 5 for $\sigma=5$ and 10 for various values of $h$. One can see from these figures that the high-frequency mode exists even at $h=0.01$. It should be noted that the existence of a weak high-frequency mode in the case of the potential (5) was first noted by Martin, Meier, and Saupe ${ }^{16}$ for the similar problem of dielectric relaxation of nematic liquid crystals.

The discussion so far has centered on the numerical solution of the problem. We shall now describe how this solution may be related to previous analytical results.
TABLE III. Product of the lowest eigenvalue $\lambda_{1}$ and the correlation time $T_{\|}$for various values of the barrier height $(\sigma)$ and field $(h)$ parameters.

\begin{tabular}{llllllll}
\hline \hline \multicolumn{10}{c}{$T_{\|} \lambda_{1}$} \\
$h$ & $\sigma=0.2$ & $\sigma=0.5$ & $\sigma=1$ & $\sigma=2$ & $\sigma=5$ & $\sigma=10$ & \multicolumn{1}{c}{$\sigma=20$} \\
\hline 0.01 & 1 & 0.999 & 0.998 & 0.994 & 0.988 & 0.996 & 0.999 \\
0.1 & 1 & 0.999 & 0.997 & 0.99 & 0.974 & 0.964 & 0.725 \\
0.2 & 1 & 0.999 & 0.995 & 0.979 & 0.896 & 0.455 & 0.00134 \\
0.4 & 1 & 0.997 & 0.986 & 0.926 & 0.332 & 0.00887 & 0.000315 \\
0.5 & 0.999 & 0.996 & 0.979 & 0.878 & 0.153 & 0.0171 & 0.00183 \\
0.7 & 0.999 & 0.992 & 0.959 & 0.746 & 0.113 & 0.0482 & 0.0176 \\
0.8 & 0.999 & 0.99 & 0.945 & 0.672 & 0.131 & 0.0695 & 0.0348 \\
1 & 0.998 & 0.984 & 0.913 & 0.544 & 0.176 & 0.119 & 0.084 \\
\hline \hline
\end{tabular}

Thus Aharoni ${ }^{4}$ has shown that for $h \leq 0.4$ and $\sigma \geq 2$ the lowest eigenvalue $\lambda_{1}$ can be approximated by Brown's formula $^{2}$

$$
\begin{aligned}
& \lambda_{1}=\pi^{-1 / 2} \sigma^{3 / 2}\left(1-h^{2}\right)\{(1+h) \exp \left[-\sigma(1+h)^{2}\right] \\
&\left.+(1-h) \exp \left[-\sigma(1-h)^{2}\right]\right\}
\end{aligned}
$$

or by noting that $\xi=2 \sigma h$ (Ref. 17):

$$
\begin{aligned}
\lambda_{1}= & 2 \pi^{-1 / 2} \sigma^{3 / 2}\left[1-\xi^{2} / 4 \sigma^{2}\right]\{\cosh \xi-(\xi / 2 \sigma) \sinh \xi\} \\
& \times \exp \left(-\sigma-\xi^{2} / 4 \sigma^{2}\right) .
\end{aligned}
$$

Thus we can also use the analytic equation (53) for evaluation of the correlation time $T_{\|}$from Eq. (4). For larger $h$ and smaller $\sigma$ this formula is inadequate (see Table III). However in this case we can use another approximate equation, which has been already derived in Ref. 11, viz., the inverse of the effective eigenvalue. ${ }^{17}$ The effective eigenvalue $\lambda_{\text {ef }}$ is determined by evaluating Eq. (17) for $l=1$ at $t=0$ :

$$
\lambda_{\mathrm{ef}}=-\frac{\dot{f}_{1}(0)}{f_{1}(0)}
$$

Thus on using Eqs. (17), (23), and (25)-(27) we can obtain an analytic equation for the effective correlation time $T_{\|}^{\mathrm{ef}}=\lambda_{\mathrm{ef}}^{-1}$ in terms of the Langevin and Dawson functions, namely ${ }^{11}$

$$
T_{\|}^{\mathrm{ef}}=2 \tau_{N} \frac{2 \sigma / T(\xi, \sigma)\left(\xi L(\xi)+1+\xi^{2} / 2 \sigma\right)-1-2 \sigma \xi^{2} / T^{2}(\xi, \sigma)}{\left.2 \sigma-2 \sigma / T(\xi, \sigma)\left[\xi L(\xi)+1-\xi^{2} / 2 \sigma\right]-\xi^{2} / 2 \sigma\right)+1}
$$

TABLE IV. Amplitudes $A_{k}$ and eigenvalues $\lambda_{k}$ of the first ten modes of the decay of the longitudinal magnetization for $h=0.01,0.2$, and 0.4 and $\sigma=10$.

\begin{tabular}{rlllllc}
\hline \hline & \multicolumn{2}{c}{$h=0.01$} & \multicolumn{2}{c}{$h=0.2$} & \multicolumn{2}{c}{$h=0.4$} \\
$k$ & \multicolumn{1}{c}{$A_{k}$} & \multicolumn{1}{c}{$\lambda_{k}$} & \multicolumn{1}{c}{$A_{k}$} & \multicolumn{1}{c}{$\lambda_{k}$} & \multicolumn{1}{c}{$A_{k}$} & $\lambda_{k}$ \\
\hline 1 & 0.858 & 0.00147 & 0.0019 & 0.0192 & $1.11 \times 10^{-6}$ & 0.19. \\
2 & $9.05 \times 10^{-8}$ & 8.06 & $2.01 \times 10^{-6}$ & 7.32 & $6.68 \times 10^{-8}$ & 7.12 \\
3 & 0.00283 & 12.3 & 0.0000632 & 12.7 & $7.86 \times 10^{-7}$ & 14.4 \\
4 & 0.000049 & 14.8 & 0.0014 & 17.6 & 0.00041 & 22.6 \\
5 & 0.000807 & 19.2 & 0.000668 & 20.3 & 0.00106 & 23.6 \\
6 & $2.18 \times 10^{-6}$ & 25.2 & 0.000101 & 26.6 & 0.000029 & 31.2 \\
7 & 0.0000361 & 32.1 & 0.0000298 & 33.4 & 0.0000227 & 37.7 \\
8 & $8.78 \times 10^{-8}$ & 40 & $5.84 \times 10^{-6}$ & 41.2 & $6.14 \times 10^{-6}$ & 44.8 \\
9 & $9.45 \times 10^{-7}$ & 49 & $1.02 \times 10^{-6}$ & 50.1 & $1.18 \times 10^{-6}$ & 53.6 \\
10 & $1.92 \times 10^{-9}$ & 58.9 & $1.5 \times 10^{-7}$ & 60 & $2.06 \times 10^{-7}$ & 63.5 \\
\hline \hline
\end{tabular}


TABLE V. Effect of the first ten modes on the decay of the magnetization for $t / \tau_{N}=0.01,0.1$, and 10 for $h=0.2$ and $\sigma=20$.

\begin{tabular}{lllll}
\hline \multicolumn{1}{c}{$t$} & \multicolumn{1}{c}{0.01} & \multicolumn{1}{c}{0.1} & \multicolumn{1}{c}{1} & 10 \\
\hline$A_{1} e^{-\lambda_{1} t}$ & $6.55 \times 10^{-7}$ & $6.55 \times 10^{-7}$ & $6.55 \times 10^{-7}$ & $6.54 \times 10^{-7}$ \\
$A_{2} e^{-\lambda_{2} t}$ & $9.66 \times 10^{-15}$ & $2.13 \times 10^{-15}$ & $5.81 \times 10^{-22}$ & $1.32 \times 10^{-87}$ \\
$A_{3} e^{-\lambda_{3} t}$ & $2.46 \times 10^{-10}$ & $2.82 \times 10^{-11}$ & $1.11 \times 10^{-20}$ & $1.03 \times 10^{-114}$ \\
$A_{4} e^{-\lambda_{4} t}$ & $2.04 \times 10^{-10}$ & $1.1 \times 10^{-11}$ & $2.27 \times 10^{-24}$ & $3.18 \times 10^{-151}$ \\
$A_{5} e^{-\lambda_{5}{ }^{t}}$ & $3.14 \times 10^{-4}$ & $6.45 \times 10^{-6}$ & $8.6 \times 10^{-23}$ & $1.53 \times 10^{-191}$ \\
$A_{6} e^{-\lambda_{6}{ }^{t}}$ & $2.22 \times 10^{-7}$ & $4.22 \times 10^{-9}$ & $2.55 \times 10^{-26}$ & $1.66 \times 10^{-198}$ \\
$A_{7} e^{-\lambda_{7} t}$ & $9.08 \times 10^{-9}$ & $5.62 \times 10^{-11}$ & $4.61 \times 10^{-33}$ & $6.36 \times 10^{-254}$ \\
$A_{8} e^{-\lambda_{8} t}$ & $8.79 \times 10^{-8}$ & $1.71 \times 10^{-10}$ & $1.29 \times 1-^{-37}$ & $8.04 \times 10^{-309}$ \\
$A_{9} e^{-\lambda_{9} t}$ & $1.29 \times 10^{-6}$ & $1.45 \times 10^{-9}$ & $4.52 \times 10^{-39}$ & $3.99 \times 10^{-334}$ \\
$A_{10} e^{-\lambda_{10} t}$ & $1.23 \times 10^{-7}$ & $6.94 \times 10^{-11}$ & $2.24 \times 10^{-43}$ & $2.77 \times 10^{-368}$ \\
\hline \hline
\end{tabular}

where the $L$ and $T$ functions are given by Eqs. (28) and (29), respectively. We compare $T_{\|}^{\text {ef }}$ and $T_{\|}$in Fig. 6 for different values of the field parameters $h$. One can see from this figure that Eq. (55) provides a good approximation to the magnetization correlation time $T_{\|}$for $h>0.5$ and all ranges of $\sigma$. This is not surprising because we have already shown ${ }^{9}$ that the effective eigenvalue method provides in general an acceptable approximation to the correlation time $T_{\|}$for $\sigma=0$ in contrast to the case $h=0$ when the effective eigenvalue approach is applicable only for low potential barriers $(\sigma<1.5)$ and there is exponentially large divergence from the exact solution in the limit of high barriers. ${ }^{5}$

The results obtained for the relaxation time are in agreement with experimental data of Barbara et al., ${ }^{18}$ where the field and temperature dependence of the relaxation time were measured for $\mathrm{Tb}_{0.5} \mathrm{Ce}_{0.5} \mathrm{Fe}_{2}$ particles. The particles had a size distribution about a mean value $150 \AA$ and cubic symmetry. Our uniaxial anisotropy model does not necessarily apply in such a case. However, it seems very likely that it should qualitatively hold

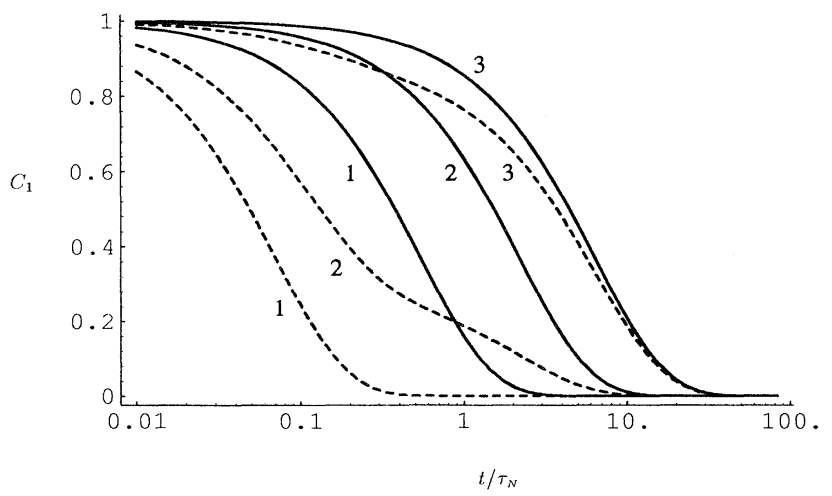

FIG. 3. Comparison of the correlation function $C_{1}(t)$ (dashed lines) with the single exponential decay function $\exp \left(-\lambda_{1} t / \tau_{N}\right)$ (solid lines) for various values of the field parameter $h$ : $0.4,0.2$, and 0.01 (curves $1-3$, respectively). for cubic anisotropy as well. ${ }^{4}$ The relaxation time $\tau$ experimentally ${ }^{18}$ measured was directly related to the reciprocal of the slope $(d M / d t)^{-1}$ of the magnetization $M(t)$ curve at $M=0$. Therefore, only the contribution of the longest lived mode to the relaxation rate was measured. Thus, we may evaluate the relaxation time $\tau$ from the equation
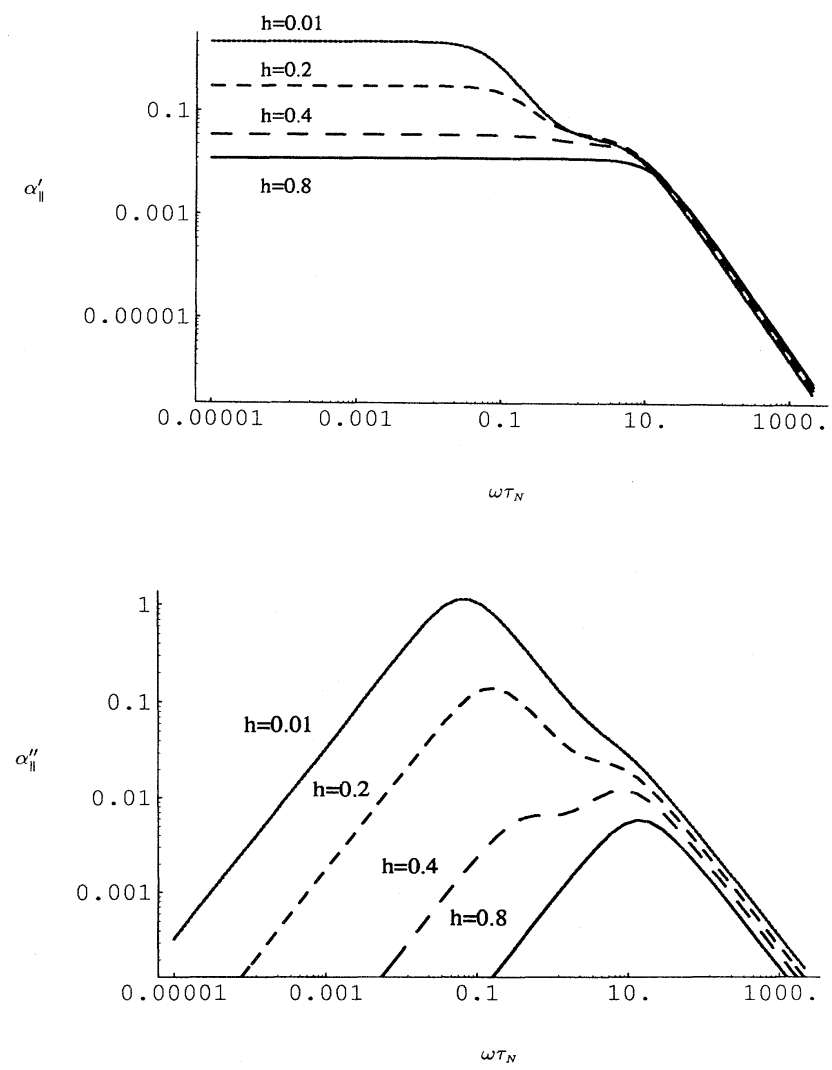

FIG. 4. The real (A) and (B) imaginary parts of the normalized susceptibility $\alpha_{\|}$as a function of frequency for $\sigma=5$ and $h=0.01,0.2,0.4$, and 0.8 . 

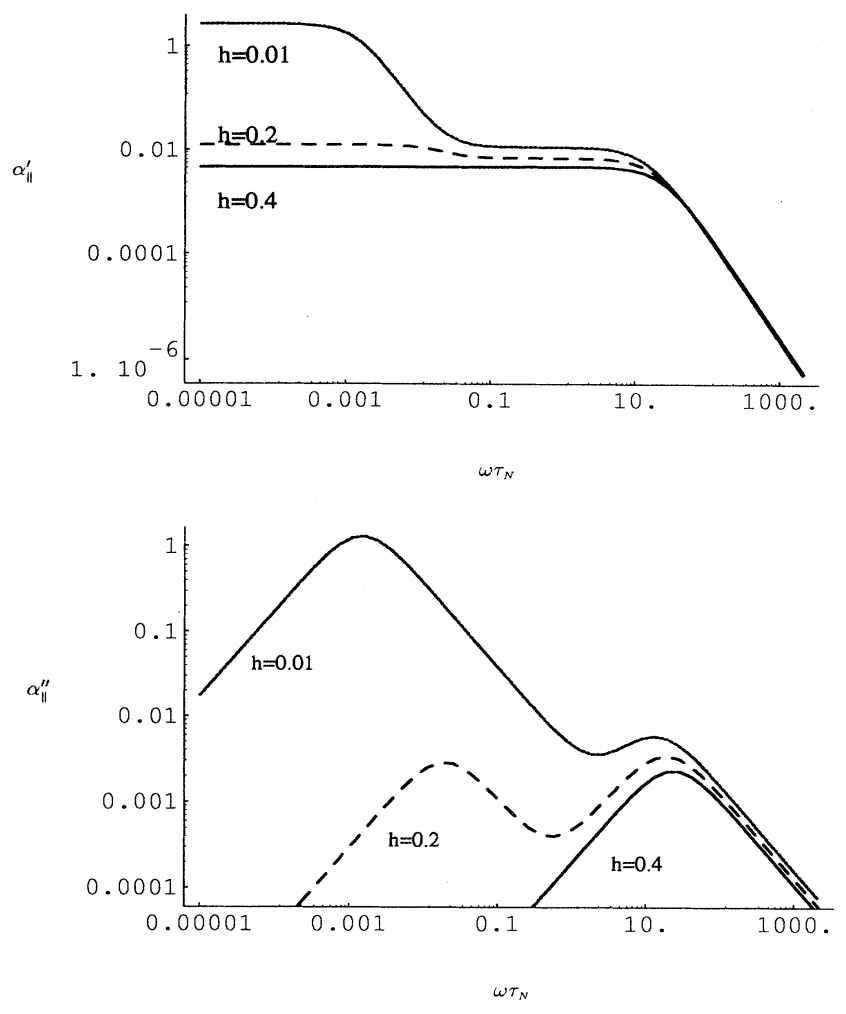

FIG. 5. The same as in Fig. 3 for $\sigma=10$ and $h=0.01,0.2$, and 0.4 .

$$
\tau=\frac{\tau_{N}}{\lambda_{1}}
$$

where $\lambda_{1}$ is given by Eq. (53). The comparison is made in Fig. 7 for two temperatures 8 and $10 \mathrm{~K}$, when the contribution of the quantum tunneling to the relaxation rate may be neglected. As observed by Barbara et al. ${ }^{18}$ the plot of $\log _{10}\left(1 / T_{\|}\right)$vs $1 / H$ at different temperatures as a parameter may be approximated by a family of straight lines with a focal point. Figure 7 shows clearly that these

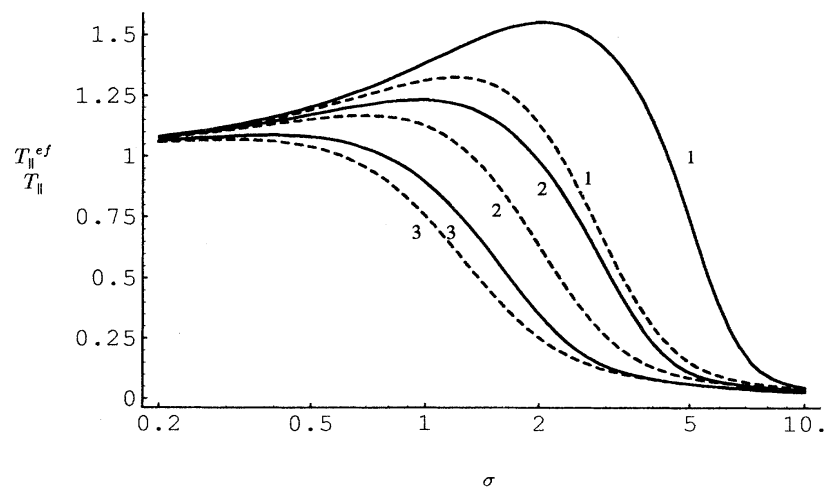

FIG. 6. Co. parison of the correlation time $T_{\|}$(solid lines) and effective correlation time $T_{\|}^{\text {ef }}$ (dashed lines) for various values of the field parameter $h=0.4,0.6$, and 1.0 (curves $1-3$, respectively).

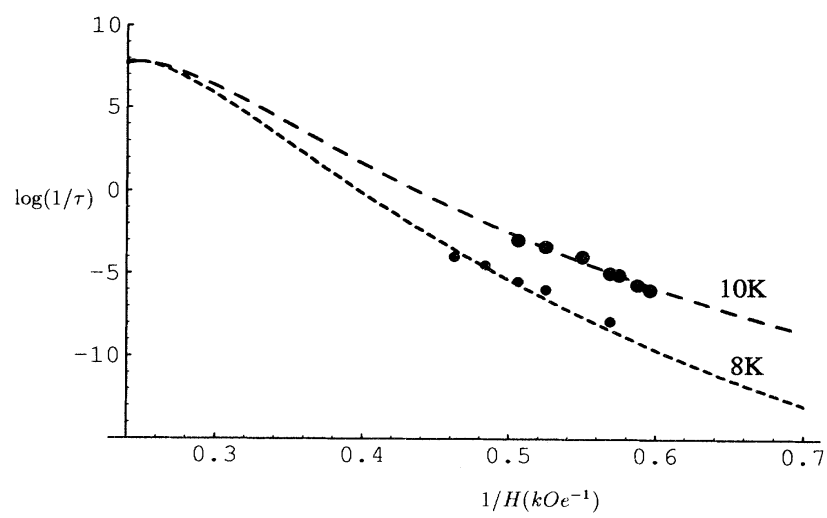

FIG. 7. Comparison of theoretical [dashed lines, Eq. (53)] and experimental [filled circles (Ref. 18)], $\log _{10}\left(1 / T_{11}\right)$ vs $1 / H$ at 8 and $10 \mathrm{~K}$ in $\mathrm{Tb}_{0.5} \mathrm{Cl}_{0.5} \mathrm{Fl}_{2}$.

observations are in qualitative agreement with the theoretical results. The values of model parameters $\sigma$ and $h$ yielded by the best fit are $\sigma=900(\mathrm{~K}) / T$ and $h=0.222\left(\mathrm{kOe}^{-1}\right) H$.

\section{CONCLUSIONS}

The analysis of Néel relaxation of single-domain magnetic particles has usually proceeded using the assumption of Brown ${ }^{2}$ that except in the very early stages of an approach to equilibrium the only appreciable timedependent term in the solution of the Fokker-Planck equation [Eq. (14)] will be that corresponding to the inverse of the lowest nonvanishing eigenvalue. The statement is certainly true in the case of zero applied field $\xi=0$. However, when $\xi \neq 0$, we have demonstrated that the higher-order decay modes have a significant role to play in the relaxation process. This effect manifests itself in two ways (i) in the large difference between $T_{\|}$and $\lambda_{1}^{-1}$ for values of $h>0.15$, (ii) in the existence of a highfrequency loss which for small values of $h$ displays itself as a shoulder in the conventional low-frequency absorption peak for $h=0$ and then predominates as $h$ increases. We remark in conclusion that we have assumed throughout (in accordance with the work described in Refs. 2 and 3 ) that the field $\mathbf{H}$ is applied along the easy axis. In practice the easy axis is in a random position so that the calculation must also be carried out when $\mathbf{H}$ is at an arbitrary angle $\psi$ to that axis. The preliminary results of such a calculation are described in Ref. 19.

\section{ACKNOWLEDGMENTS}

We would like to thank the British Council, FORBAIRT and the Trinity College Dublin Trust for grants in support of this work. D.S.F.C. acknowledges UK-SERC Rolling Grant No. GR/H59862. Yu.P.K. gratefully acknowledges the Royal Irish Academy and the Research and Postgraduate Studies Committee of Dublin City University for financial support. We would also like to thank Professor J. M. D. Coey for a helpful conversation. 
${ }^{*}$ Author to whom correspondence should be sent. Electronic address: secr@mee.tcd.ie

${ }^{1}$ L. Néel, Ann. Géophys. 5, 99 (1949).

${ }^{2}$ W. F. Brown, Jr., Phys. Rev. 130, 1677 (1963).

${ }^{3}$ A. Aharoni, Phys. Rev. 135A, 447 (1964).

${ }^{4}$ A. Aharoni, Phys. Rev. 177, 793 (1969).

${ }^{5}$ W. T. Coffey, D. S. F. Crothers, Yu. P. Kalmykov, E. S. Massawe, and J. T. Waldron, Phys. Rev. E 49, 1869 (1994).

${ }^{6}$ H. A. Kramers, Physica 7, 248 (1940).

${ }^{7}$ D. A. Garanin, V. V. Ischenko, and L. V. Panina, Teor. Mat. Fiz. 82, 242 (1990) [Theor. Math. Phys. 82, 169 (1990)].

${ }^{8}$ W. T. Coffey, Yu. P. Kalmykov, E. S. Massawe, and J. T. Waldron, J. Chem. Phys. 99, 4011 (1993).

${ }^{9}$ J. T. Waldron, Yu. P. Kalmykov, and W. T. Coffey, Phys. Rev. E 49, 3976 (1994).

${ }^{10}$ W. T. Coffey, Yu. P. Kalmykov, and J. T. Waldron, Physica A 208, 462 (1994).

${ }^{11}$ W. T. Coffey, P. J. Cregg, and Yu. P. Kalmykov, Adv. Chem. Phys. 83, 263 (1993).
${ }^{12}$ Handbook of Mathematical Functions, edited by $\mathbf{M}$. Abramowitz and I. Stegun (Dover, New York, 1964).

${ }^{13} \mathrm{H}$. Risken, The Fokker-Planck Equation (Springer, Berlin, 1984).

${ }^{14}$ M. Krasnov, A. Kiselev, G. Makarenko, and E. Shikin, Mathematical Analysis for Engineers (Mir, Moscow 1990), Vol. 2.

${ }^{15}$ B. K. P. Scaife, Principles of Dielectrics (Oxford University, Oxford, 1989).

${ }^{16}$ A. J. Martin, G. Meier, and S. Saupe, Symp. Faraday Soc. 5, 119 (1971).

${ }^{17}$ W. T. Coffey, Yu. P. Kalmykov, and E. S. Massawe, Adv. Chem. Phys. 85, (part 2), 667 (1993).

${ }^{18}$ B. Barbara, L. C. Sampaio, J. E. Wegrowe, B. A. Ratnam, A. Marchand, C. Paulsen, M. A. Novak, J. L. Tholence, M. Uehara, and D. Fruchart, J. Appl. Phys. 73, 6703 (1993).

${ }^{19}$ W. T. Coffey, D. S. F. Crothers, J. L. Dormann, L. J. Geoghegan, Yu. P. Kalmykov, J. T. Waldron, and A. W. Wickstead, J. Magn. Magn. Mater. 145, L263 (1995). 